\title{
Magnetic Resonance Imaging of the Phase Separation in Mixed Preparations of Moisturizing Cream and Steroid Ointment after Centrifugation
}

\author{
Yoshinori Onuki, ${ }^{* a, \dagger}$ Chiaki Funatani, ${ }^{a}$ Takashi Yokawa, ${ }^{b}$ Yoshihisa Yamamoto, ${ }^{c}$ Toshiro Fukami, ${ }^{d}$ \\ Tatsuo Koide, ${ }^{e}$ Yasuko Obata, ${ }^{a}$ and Kozo Takayama ${ }^{a}$ \\ ${ }^{a}$ Department of Pharmaceutics, Hoshi University; 2-4-41 Ebara, Shinagawa-ku, Tokyo 142-8501, Japan: ${ }^{b}$ Bio \\ View Corporation; 2-16-16 Iwamoto-cho, Chiyoda-ku, Tokyo 101-0032, Japan: ${ }^{c}$ Faculty of Pharmaceutical \\ Sciences, Teikyo Heisei University; 4-21-2 Nakano, Nakano-ku, Tokyo 164-8530, Japan: ${ }^{d}$ Department of Molecular \\ Pharmaceutics, Meiji Pharmaceutical University; 2-522-1 Noshio, Kiyose, Tokyo 204-8588, Japan: and ${ }^{e}$ Division of \\ Drugs, National Institute of Health Sciences; 1-18-1 Kamiyoga, Setagaya-ku, Tokyo 158-8501, Japan. \\ Received January 26, 2015; accepted February 16, 2015
}

A mixed preparation consisting of a water-in-oil emulsion-type moisturizing cream and a steroid ointment is frequently prescribed for the treatment of atopic dermatitis. We have investigated the compatibility of moisturizing creams and ointments because there are concerns regarding the physical stability of these mixed preparations. The key technology used in this study was magnetic resonance imaging (MRI). A commercial moisturizing cream and white petrolatum or clobetasone butyrate (CLB) ointment samples were mixed in a weight ratio of $1: 1$. A centrifugation test protocol $(20000 \times g$ for $3 \mathrm{~min})$ was implemented to accelerate the destabilization processes in the samples. After centrifugation, the mixed preparations separated into three distinct layers (upper, middle, and lower), while no phase separation was observed using moisturizing cream alone. The phase separation was monitored using chemical shift selective images of water and oil and quantitative $T_{2}$ maps. In addition, MR and near-infrared spectroscopy were employed for component analysis of each phase-separated layer. Collectively, it was confirmed that the lower layer contained water, oils, and organic solvent, while the upper and middle layers were composed solely of oils. Furthermore, this study investigated the distribution of CLB in the phase-separated samples and showed that a heterogeneous distribution existed. From our results, it was confirmed that the mixed preparation became unstable because of the incompatibility of the moisturizing cream and ointment.

Key words mixed external preparation; magnetic resonance (MR) spectroscopy; MR imaging (MRI); chemical shift selective image; quantitative $T_{2}$ map; clobetasone butyrate ointment

In the treatment of atopic dermatitis, moisture retention of the skin is regarded as being important, in addition to steroid therapy. From this perspective, a combination therapy containing a steroid ointment and a moisturizing cream is widely prescribed to patients. To make the application of these preparations easier, pharmacists frequently mix moisturizing cream with a steroid ointment when preparing the prescription.

Nowadays, numerous moisturizing creams are available from several manufacturers. Water-in-oil (w/o) emulsion-type creams are regarded as being the most suitable for preparing mixed preparations because the oily continuous phase is assumed to be miscible with the ointment. However, it is doubtful whether these pharmaceutical emulsions remain stable after being mixed with other preparations. Their formulations were not designed under the assumption that they would be mixed with other preparations, and by mixing these formulations with other preparations the optimal hydrophile-lipophile balance (HLB) and surfactant content necessary to maintain a stable dispersion state may be changed. Four distinct destabilization processes of emulsions are known: creaming, flocculation, coalescence, and Ostwald ripening. ${ }^{1)}$ Coalescence and Ostwald ripening are the most serious types of destabilization,

\footnotetext{
†Present address: Department of Pharmaceutical Technology, Graduate School of Medicine and Pharmaceutical Science for Research, University of Toyama; 2630 Sugitani, Toyama 930-0194, Japan.
}

while creaming and flocculation are subtle destabilization processes. These processes are not independent, and each one can influence or be influenced by the other processes. In practice, they may proceed simultaneously or occur in any order. The subtle destabilization processes are potential steps towards more serious destabilization, and thus the subtle destabilization processes, creaming and flocculation, are key criteria for evaluating the physical stability of pharmaceutical emulsions.

To date, various methods have been proposed to evaluate the physical stability of emulsions. These include droplet size analysis, ${ }^{2,3)}$ light scattering, ${ }^{2,4,5)}$ and turbidity measurements. ${ }^{6}$ ) However, the sensitivity of these techniques is insufficient to detect the subtle destabilization processes of pharmaceutical emulsions. In addition, these sampling techniques are destructive by nature, and samples need be transferred to an appropriate sample container (e.g., a transparent cuvette or tube) for the measurements to take place. Thus, we cannot elucidate the natural destabilization processes of pharmaceutical emulsions in a commercial container (e.g., a tube or jar). Given these issues, there is a great demand to develop a nondestructive method that would enable the detection of creaming or flocculation in pharmaceutical emulsions.

Recently, we have applied magnetic resonance imaging (MRI) to evaluate the physical stability of pharmaceutical emulsions. ${ }^{7,8)}$ MRI is one of the most popular molecular imaging methods, and enables nondestructive monitoring of 
a sample using the principle of nuclear magnetic resonance (NMR). In addition, it can visualize the molecular mobility of a sample using magnetic resonance (MR) parameters. In a previous study, we succeeded in visualizing creaming in model pharmaceutical emulsions. The essential element of our novel method is the visualization of the state of water. ${ }^{7)}$ It is also worth noting that the sensitivity of the detection limit of our method is superior to that of visible observations; for example, slight creaming, which is not visible to the naked eye, can be visualized clearly.

In this study, we investigated the physical stability of mixed preparations. The test samples were prepared by mixing a commercial moisturizing cream with either a white petrolatum or a clobetasone butyrate (CLB) ointment. The mixed preparations were centrifuged to accelerate the destabilization processes. We made the most of the MR technique to evaluate the compatibility of the moisturizing cream and ointments. As well as acquiring MR images using a method that we have previously employed, ${ }^{7}$ in this study, we acquired chemical shift selective (CSS) images to visualize the distribution of water and oils in the samples. The images were constructed by exciting protons in a very narrow frequency range, which enabled us to visualize the spatial distribution of the substance of interest in the samples. Using the CSS images, we succeeded in visualizing the heterogeneous distributions of water and oil in the phase-separated samples. In addition, we performed component analysis using MR spectroscopy (MRS) and near-infrared (NIR) spectroscopy. From this study, we have achieved a comprehensive understanding of the physical stability of mixed preparations for external use.

\section{Experimental}

Materials The w/o-type moisturizing cream used (Hildoid $^{\circledR}$ soft ointment $0.3 \%$ ) was purchased from Maruho Co., Ltd. (Osaka, Japan). The CLB ointment used (Kindavate ${ }^{\circledR}$ ointment) was purchased from GlaxoSmithKline KK (Tokyo, Japan). The white petrolatum used (Sun white P-150) was purchased from Nikko Rika (Tokyo, Japan). The liquid paraffin used was purchased from Wako Pure Chemical Industries, Ltd. (Osaka, Japan). The CLB used was purchased from Sigma-Aldrich Chemicals (St. Louis, MO, U.S.A.). All other chemicals used were of analytical grade.

MRI Study For sample preparation, the moisturizing cream was mixed with an equal volume of white petrolatum for 3 min using a revolution/rotation-type hybrid mixer (Model HM-500, Keyence, Tokyo, Japan). The revolution and rotation speeds were 2000 and $800 \mathrm{rpm}$, respectively. The moisturizing cream alone was also investigated as a reference sample. To accelerate the destabilization processes in the samples, a centrifugation test protocol was performed whereby the sample was placed in centrifuge tubes and then centrifuged in a Model MA-2024 rotor (Kubota, Tokyo, Japan) at $20000 \times \boldsymbol{g}$ for $3 \mathrm{~min}$. The sample was stored at room temperature until used in the MRI experiments.

The MRI experiments were performed at room temperature using a 9.4T vertical MRI scanner (Varian, Palo Alto, CA, U.S.A.). The ${ }^{1} \mathrm{H}-\mathrm{NMR}$ spectrum of the entire sample was acquired using an S-PLUS sequence, while the spectrum of a region of interest (ROI) was acquired using a stimulated echo acquisition mode (STEAM) sequence (acquisition bandwidth $=5 \mathrm{kHz}$ ). The $T_{1}$-weighted images ( $T_{1}$ WIs) were acquired using a gradient echo pulse sequence with repetition time $(\mathrm{TR})=12 \mathrm{~ms}$, echo time $(\mathrm{TE})=6 \mathrm{~ms}$, field of view $(\mathrm{FOV})=30 \times 30 \mathrm{~mm}^{2}$, matrix size $=256 \times 256$, number of excitations $=2$, slice thickness $=1 \mathrm{~mm}$, and a flip angle of $10^{\circ}$. A $T_{2}$ weighted image $\left(T_{2} \mathrm{WI}\right)$ was acquired using a spin-echo pulse sequence with $\mathrm{TR}=2000 \mathrm{~ms}, \mathrm{TE}=40 \mathrm{~ms}, \mathrm{FOV}=30 \times 30 \mathrm{~mm}^{2}$, matrix size $=128 \times 128$, number of excitations $=2$, and slice thickness $=1 \mathrm{~mm}$. In addition, two other images with TE values of 25 and $60 \mathrm{~ms}$ were acquired, and then a quantitative $T_{2}$ map was constructed from the three images. The CSS images were acquired using a CSS spin-echo pulse sequence comprising $90^{\circ}$ and $180^{\circ}$ Gaussian radio frequency (RF) pulses. The $90^{\circ}$ pulse was band selective and the $180^{\circ}$ pulse was a sliceselective refocusing pulse. We set the center frequency for the water and hydrocarbon protons from the ${ }^{1} \mathrm{H}-\mathrm{NMR}$ spectra acquired using the S-PLUS sequence. The bandwidth of the RF pulses was $540 \mathrm{~Hz}$. All the CSS images were acquired using the following parameters: $\mathrm{TR}=2000 \mathrm{~ms}, \mathrm{TE}=15.17 \mathrm{~ms}$, $\mathrm{FOV}=30 \times 30 \mathrm{~mm}^{2}$, matrix size $=128 \times 128$, number of excitations $=1$, and slice thickness $=1 \mathrm{~mm}$.

Measurement of NIR Spectra Mixed preparations consisting of the moisturizing cream and white petrolatum were prepared and centrifuged in the same manner as described above, and then small samples of each phase-separated layer were collected to acquire the NIR spectra. The acquisition of NIR spectra was determined using a Spectrum One NTS spectrometer (PerkinElmer, Inc., Waltham, U.S.A.) equipped with an Omni Cell system used for mulls (Specac Inc., Cranston, U.S.A.) at a wavenumber resolution of $4 \mathrm{~cm}^{-1}$ employing 32 scans across the wavelength range $4000-8000 \mathrm{~cm}^{-1}$. Liquid paraffin, white petrolatum and the moisturizing cream was also investigated as a reference sample. These experiments also acquired the NIR spectra of air as a background.

Quantification of CLB in the Phase-Separated Layers of Centrifuged Samples For preparing these samples, instead of white petrolatum, CLB ointment $(500 \mu \mathrm{g} / \mathrm{g})$ was mixed with the moisturizing cream in a weight ratio of $1: 1$ using a hybrid mixer, and then the centrifugation test protocol $(20000 \times \boldsymbol{g}$ for $3 \mathrm{~min}$ ) was performed. CLB was extracted from the samples according to the method used in our previous study with minor modifications. ${ }^{9)}$ In brief, small samples were obtained from the upper, middle, and lower layers. These were dispersed in a designated volume of hexane, and then a volume of 1.67 times the sample volume of acetonitrile was added to the mixture, which was then shaken for $10 \mathrm{~min}$. The quantification of CLB was performed using high-performance liquid chromatography (HPLC). The sample solution was injected into a Hitachi L-2130 HPLC pump equipped with a C18 reversedphase column (YMC-pack Model ODS-A, ID $=150 \times 4.6 \mathrm{~mm}^{2}$, Yamamura Chemical Laboratories, Kyoto, Japan). A Hitachi UV detector Model L-2400 (Tokyo, Japan) was set at a wave-

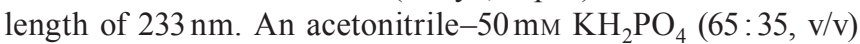
solution was used as the mobile phase, the flow rate used was $1.0 \mathrm{~mL} / \mathrm{min}$, and the HPLC analysis was performed at room temperature. An EZChrom Elite ${ }^{\circledR}$ Chromatography Data System (Hitachi, Tokyo, Japan) was used as the acquisition and analysis software system. The mixed preparation without the centrifugation protocol (homogeneous sample, CLB concentration of $250 \mu \mathrm{g} / \mathrm{g}$ ) was also investigated as a control. The concentrations of CLB in the phase-separated layers were calculated relative to the peak area of the control. 
(a)

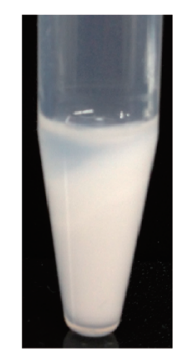

(b)
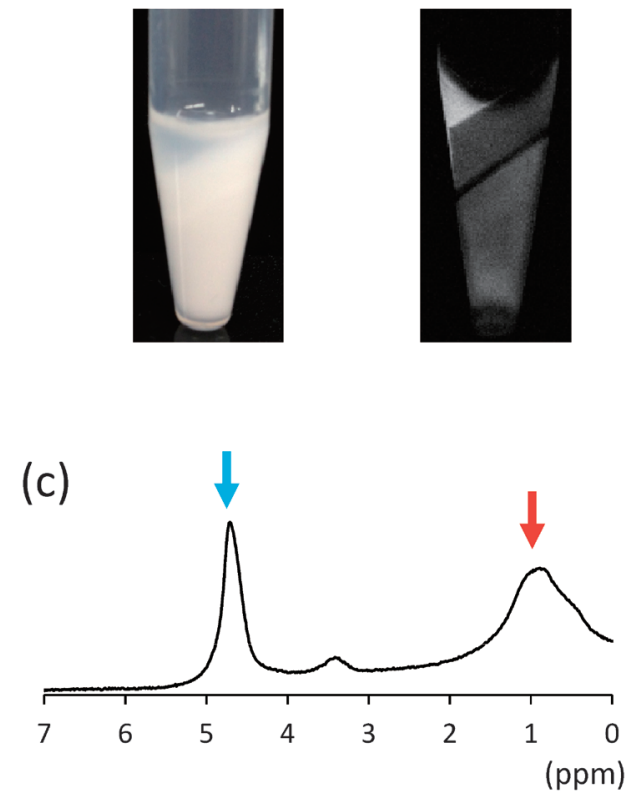

(d)

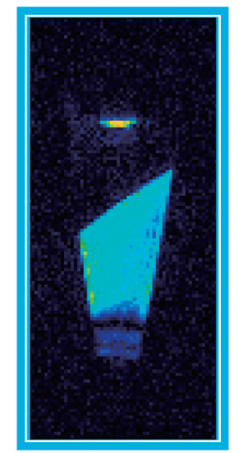

(e)

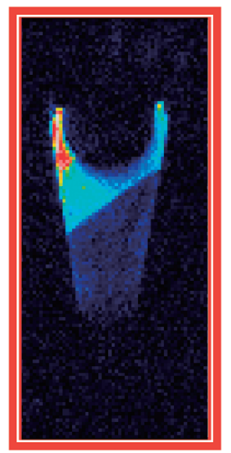

Fig. 1. Nondestructive Monitoring of a Mixed Preparation after Centrifugation

The moisturizing cream was mixed with white petrolatum in a weight ratio of $1: 1$, and then centrifuged at $20000 \times \boldsymbol{g}$ for $3 \mathrm{~min}$. The centrifuged sample was monitored using (a) optical photography and (b) $T_{1}$ WI. (c) The ${ }^{1} \mathrm{H}-\mathrm{NMR}$ spectrum of the entire sample acquired using S-PLUS. The acquisition parameters were $\mathrm{TR}=2000 \mathrm{~ms}$ and $\mathrm{TE}=11.7 \mathrm{~ms}$ from an average of 128 scans. (d) Water and (e) oil images acquired using CSS pulse sequence.

\section{Results}

Nondestructive Monitoring of the Samples Using MRI

Figures $1 \mathrm{a}$ and $\mathrm{b}$ show the visible observation and $T_{1} \mathrm{WI}$ of the mixed preparations consisting of moisturizing cream and white petrolatum. It can be seen that the mixed preparations separated into three distinct layers after centrifugation. The upper layer was relatively clear and a leveling of the surface was observed at the top of the samples, indicating that the layer was in the liquid state. The middle and lower layers seemed to be more rigid than the upper layer, and a surface gradient between the middle and lower layers remained after centrifugation.

Figure 1c shows the ${ }^{1} \mathrm{H}-\mathrm{NMR}$ spectrum obtained from the entire sample. Two main peaks occurring at $1.3 \mathrm{ppm}$ (red arrow) and $4.7 \mathrm{ppm}$ (blue arrow) were observed in the spectrum. These were assigned to the hydrocarbon groups of the oils and to water, respectively. A small peak was also (a)

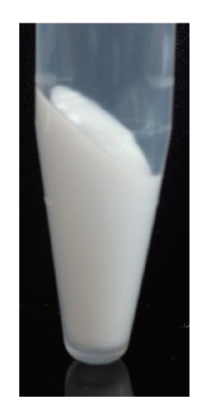

(b)
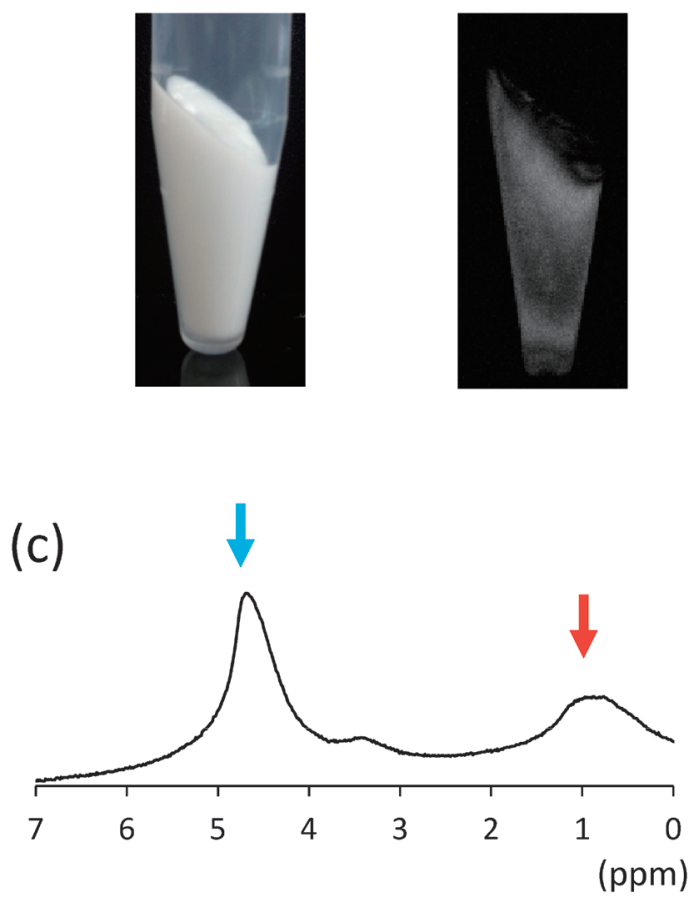

(d)

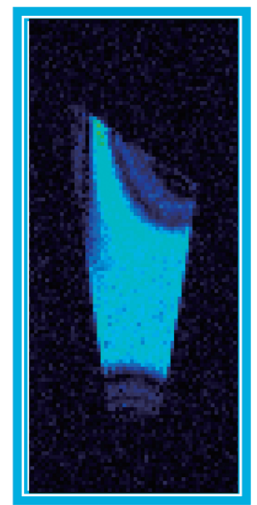

(e)

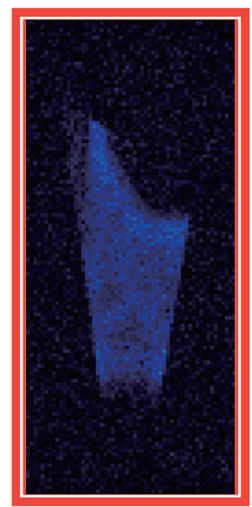

Fig. 2. Nondestructive Monitoring of the Original Moisturizing Cream after Centrifugation

The moisturizing cream alone was centrifuged at $20000 \times \boldsymbol{g}$ for $3 \mathrm{~min}$. The centrifuged sample was monitored using (a) optical photography and (b) $T_{1}$ WI. (c) The ${ }^{1} \mathrm{H}-\mathrm{NMR}$ spectrum of the entire sample acquired using S-PLUS. The acquisition parameters were $\mathrm{TR}=2000 \mathrm{~ms}$ and $\mathrm{TE}=11.7 \mathrm{~ms}$ from an average of 128 scans. (d) Water and (e) oil images acquired using CSS pulse sequence.

observed at $3.3 \mathrm{ppm}$, which probably arises from the hydroxyl groups of the hydrophilic organic solvents, such as glycerin. Because there was a sufficient difference in the chemical shift of the substances to be differentiated, the water and oil protons were selectively excited using a CSS pulse sequence to create their own images (Figs. 1d, e).

The CSS images clearly showed the differences in the distribution between the water and the oil in the phase-separated samples. The water seemed to be located only in the lower layer, while most of the oil was distributed in the upper and middle layers, with only some oil being incorporated into the lower layer. Furthermore, in the oil images, the contrast in the upper layer was much more intense than that in the middle 
layer, suggesting that the oils in the upper layer were different from those in the middle layer.

Regarding the moisturizing cream alone, no phase separation was observed in either visible observations or the $T_{1} \mathrm{WI}$ data (Figs. 2a, b). As shown in the ${ }^{1} \mathrm{H}-\mathrm{NMR}$ spectrum (Fig. $2 \mathrm{c}$ ), the intensity of the hydrocarbon peak occurring at $1.3 \mathrm{ppm}$ was lower than that shown in Fig. 1c, because this sample was not mixed with any white petrolatum; however, it was still possible to observe this peak, we therefore created both water and oil images (Figs. 2d, e). These images showed that the distributions of water and oil were fully consistent with each other. Taken together, it was confirmed that the original pharmaceutical emulsion was much more stable against centrifugation than the mixed preparation.

Component Analysis of the Phase-Separated Layers from the ${ }^{1} \mathbf{H}$-NMR and NIR Spectra The ${ }^{1} \mathrm{H}-\mathrm{NMR}$ spectra of the phase-separated layers were acquired using MRS. The ROIs were positioned in the center of each layer. The voxel size of the ROIs was fixed at $1 \times 1 \times 1 \mathrm{~mm}^{3}$. These spectra clearly showed that the composition of the lower layer was different from those of the middle and upper layers (Fig. 3). From the spectrum of the lower layer, peaks occurring at 1.3, 3.3, and $4.7 \mathrm{ppm}$ were observed, and as discussed, these denote the hydrocarbon groups of the oils, the hydrophilic groups of the organic solvent, and the water protons, respectively. In contrast, in the spectra from the upper and middle layers, only hydrocarbon group peaks occurring around $1.3 \mathrm{ppm}$ were observed, and the spectral patterns from the two layers resembled each other.

Next, we acquired NIR spectra from each layer (Fig. 4). The results were in good agreement with the results obtained
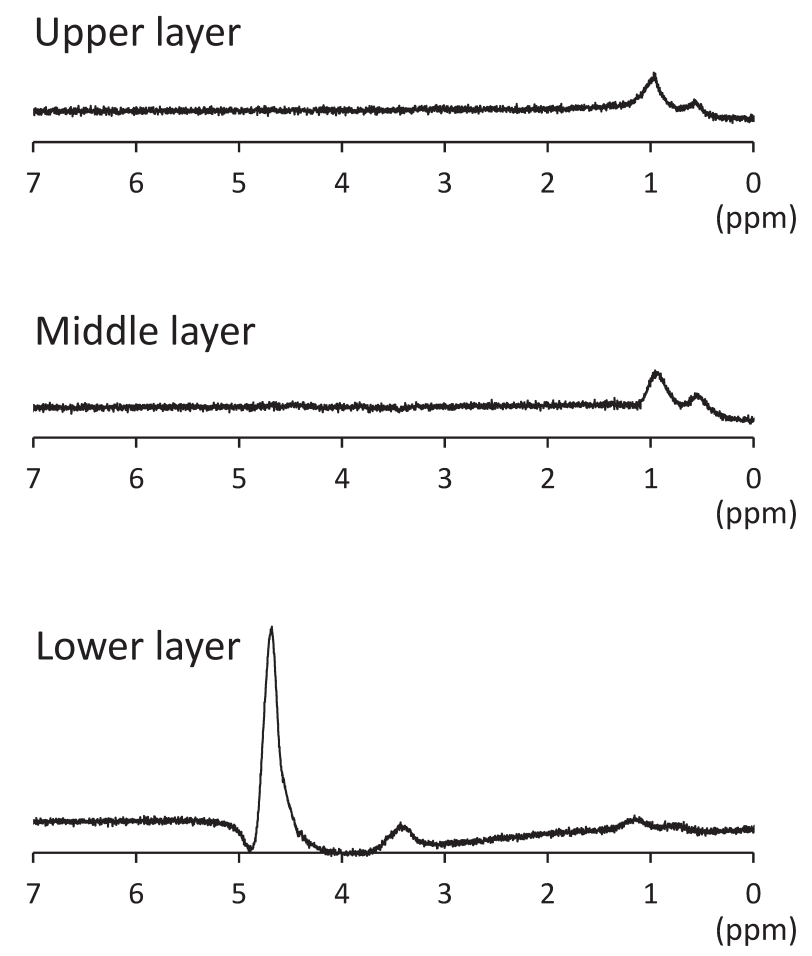

Fig. 3. ${ }^{1} \mathrm{H}-\mathrm{NMR}$ Spectra of Each Phase-Separated Layer Acquired Using STEAM

The moisturizing cream was mixed with white petrolatum in a weight ratio of $1: 1$, and then centrifuged at $20000 \times \boldsymbol{g}$ for $3 \mathrm{~min}$. ROIs $\left(1 \times 1 \times 1 \mathrm{~mm}^{3}\right)$ were positioned in the center of the upper, middle, and lower layers. The acquisition parameters were $\mathrm{TR}=2000 \mathrm{~ms}$ and $\mathrm{TE}=11.7 \mathrm{~ms}$ from an average of 128 scans. from the MRS data. First, peaks occurring at $5200 \mathrm{~cm}^{-1}$ and $7000 \mathrm{~cm}^{-1}$ were assigned to a combination and to the first overtone bands of hydroxyl $(\mathrm{O}-\mathrm{H})$ group, respectively. These peaks are mostly derived from water in the sample. Intense water peaks were detected in the lower layer, and a weak peak occurring at $5200 \mathrm{~cm}^{-1}$ was also observed in the middle layer. In contrast, no water peaks were observed in the upper layer. As the spectra of white petrolatum and liquid paraffin showed peaks occurring at $4000-4500 \mathrm{~cm}^{-1}$ and $5500-6000 \mathrm{~cm}^{-1}$, these were assigned to the combination and to the first overtone bands of hydrocarbons (i.e., $\mathrm{C}-\mathrm{H}$ groups). Hydrocarbon peaks were observed in the spectra from all layers. As in the ${ }^{1} \mathrm{H}-\mathrm{NMR}$ spectra, it was difficult to find differences between the upper and middle layers from the spectral patterns. For further information, the spectra of the lower layer well agreed with that of the original moisturizing cream.

In summary, it was concluded that the lower layer is an aqueous layer containing a considerable amount of the oils and hydrophilic organic solvent, while the upper and middle layers were only composed of oils.

Quantitative $T_{2}$ Map of the Phase-Separated Samples We acquired $T_{2}$ maps of the phase-separated samples (Fig. 5a). The $T_{2}$ values of the layers seemed to be different, and so histograms of the $T_{2}$ values of the layers were generated from the maps (Fig. 5b). The mean values of $T_{2}$ were 27.34, 18.35, and $20.47 \mathrm{~ms}$ for upper, middle, and lower layers, respectively. It is worth noting that despite the similar patterns in the ${ }^{1} \mathrm{H}-\mathrm{NMR}$ and NIR spectra (Figs. 3, 4), the components of the upper and middle layers were different in terms of the $T_{2}$ values (Fig. 5 b): the $T_{2}$ value of the upper layer was longer than that of the middle layer.

CLB Concentration in the Phase-Separated Layers of the Centrifuged Samples We investigated the distribution of CLB in the mixed preparations occurring during phase separation. The concentration of CLB in each phase-separated layer was obviously different: the CLB concentrations in the upper, middle, and lower layers were $156.0 \pm 6.9,136.4 \pm 3.1$, and $397.5 \pm 2.8 \mu \mathrm{g} / \mathrm{g}$, respectively (Fig. 6).

\section{Discussion}

First, we monitored the phase separation in a mixed preparation consisting of moisturizing cream and white petrolatum. The phase-separated layers seemed to be different from each other in terms of their appearance and texture (Figs. 1a, b). Then, we visualized the distribution of water and oil in the phase-separated sample using CSS images. ${ }^{10)}$ In general, MR images are constructed by summing the NMR signals from all liquid state protons irrespective of their chemical structure, and so MRI data usually lack the specific spectroscopic information contained within the NMR signals. ${ }^{11)}$ On the other hand, CSS images are constructed by selectively exciting a targeted proton, and thus enable us to visualize the spatial distribution of a compound of interest. ${ }^{10)}$ From this perspective, CSS images can be referred to as an imaging technique that integrates MRS data into MRI data. In addition, CSS images have the capacity to monitor a large area with a higher spatial resolution. To date, CSS images have been applied to visualize the spatial distribution of various compounds, and have been used to quantify them. These compounds include water, ${ }^{12-14)}$ hydrocarbon groups in fats and oils, ${ }^{13-17)}$ aromatic compounds, ${ }^{17)}$ and sucrose. ${ }^{18)}$ To the best of our knowledge, 


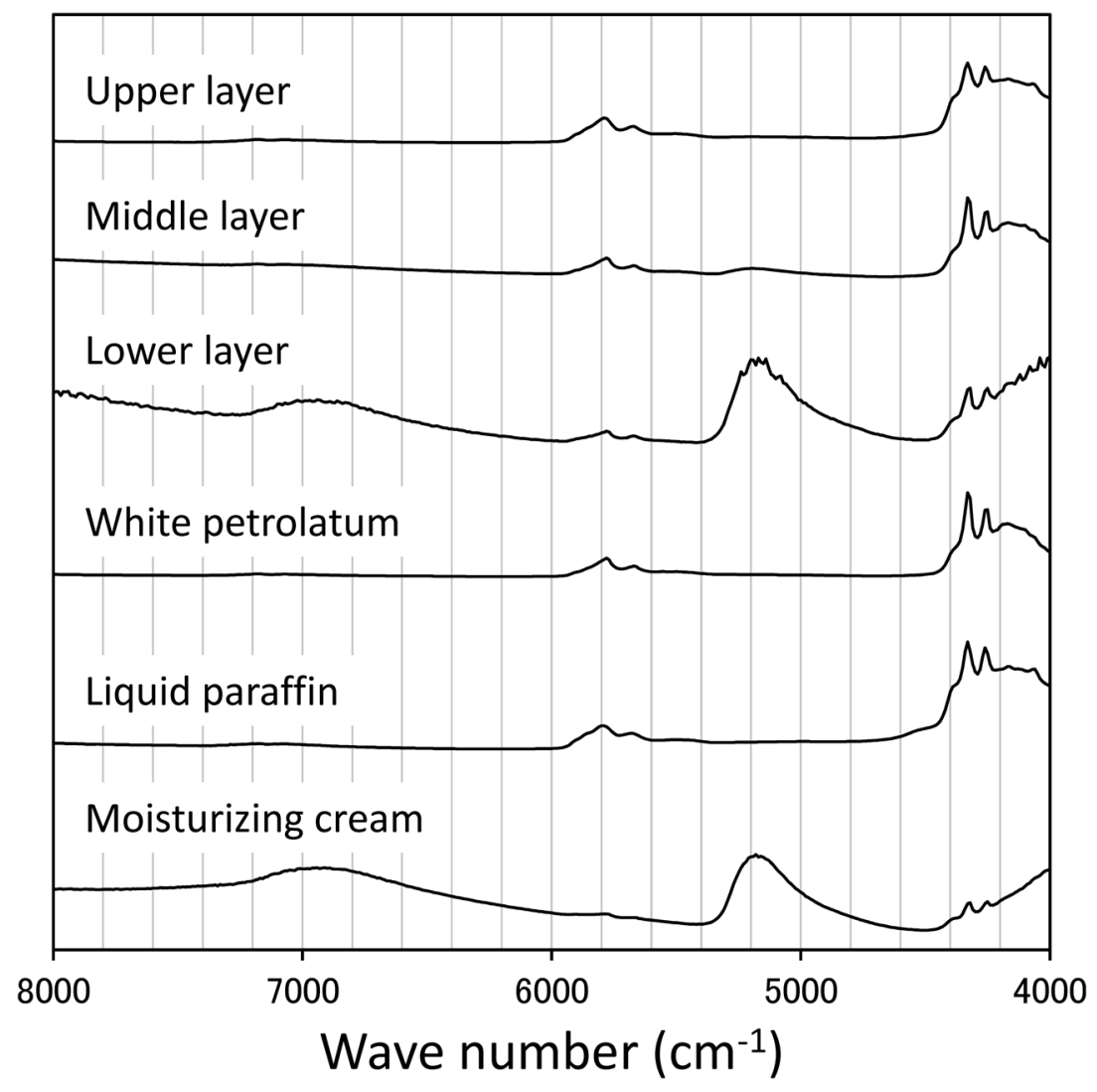

Fig. 4. NIR Spectra of Each Phase-Separated Layer

The moisturizing cream was mixed with white petrolatum in a weight ratio of $1: 1$ and then centrifuged at $20000 \times \boldsymbol{g}$ for 3 min. Small samples were collected from each layer and their NIR spectra were acquired. Liquid paraffin, white petrolatum and the moisturizing cream was also investigated as a reference sample.

(a)
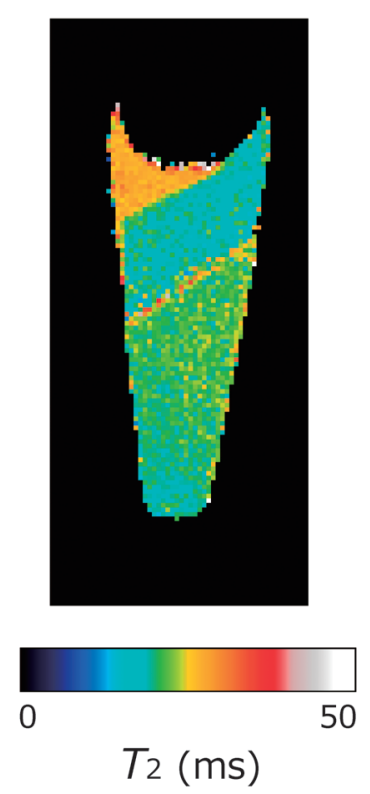

(b)

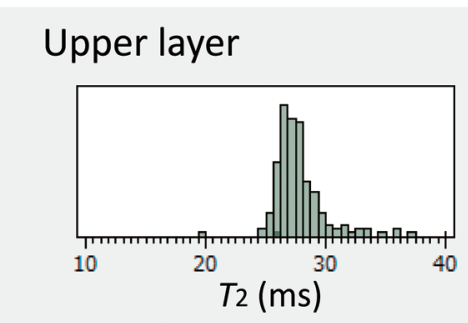

\section{Middle layer}

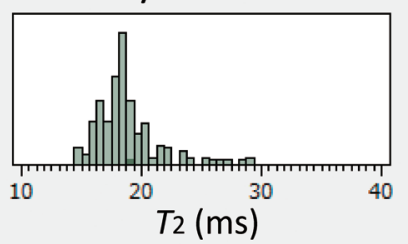

\section{Lower layer}

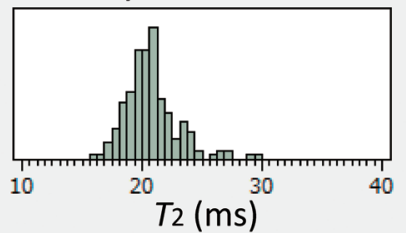

Fig. 5. Visualization of the Molecular State of a Phase-Separated Sample

The moisturizing cream was mixed with white petrolatum in a weight ratio of $1: 1$ and then centrifuged at $20000 \times \boldsymbol{g}$ for 3 min. The centrifuged sample was monitored by quantitative $T_{2}$ map (a). (b) Histograms of $T_{2}$ were generated by randomly sampling $T_{2}(N=200)$ from each phase-separated layer. 


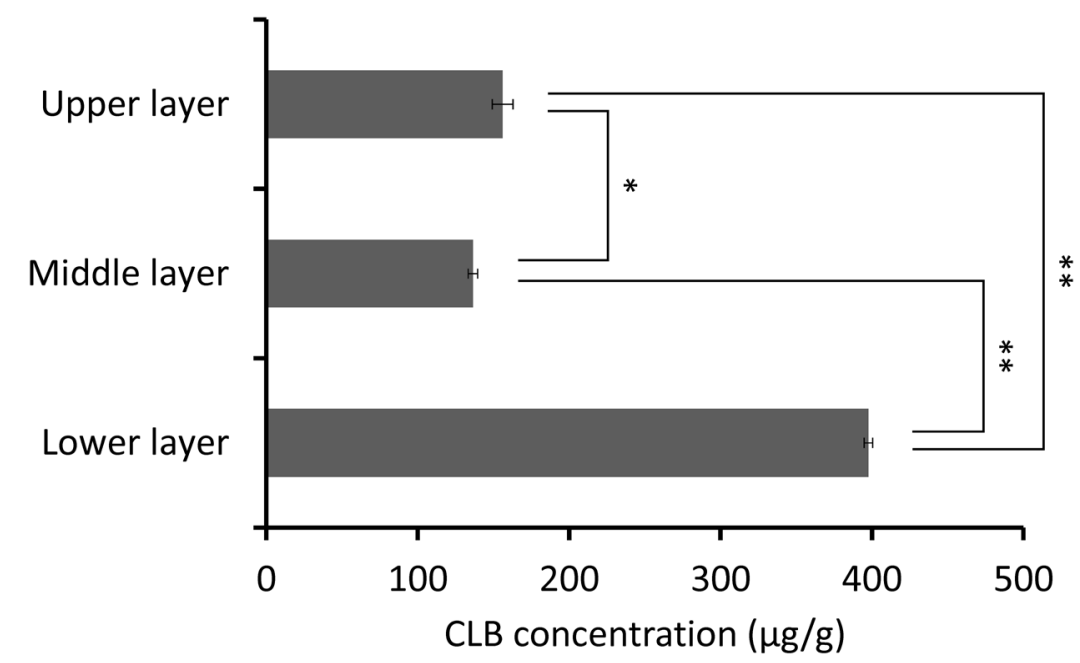

Fig. 6. The CLB Concentration in Each Phase-Separated Layer

The moisturizing cream was mixed with CLB ointment in a weight ratio of $1: 1$ and then centrifuged at $20000 \times \mathbf{g}$ for 3 min. CLB incorporated into the layers was extracted and then quantified using HPLC. Each bar denotes the mean value \pm S.D. $(N=3)$. ${ }^{*} p<0.05$ and ${ }^{* *} p<0.01$ (Tukey-Kramer's method).

this paper is the first technical report of the application of CSS images to evaluate the physical properties of pharmaceutical emulsions. From the CSS images (Figs. 1d, e), water seemed to be located in only the lower layer, while oils were mostly located in the upper and middle layers, with a part of the oils located in the lower layer.

We also investigated the moisturizing cream alone. A series of experiments showed that the original pharmaceutical emulsion was stable against centrifugation (Fig. 2). No phase separation was observed in the centrifuged samples and the distribution of water and oil were fully consistent with each other. These results indicate that the physical stability of the mixed preparations decreased substantially on mixing the moisturizing cream with an oily ointment base.

In the next phase of the study, we performed component analysis of the phase-separated samples using MRS and NIR spectroscopy. MRS is a technique that enables the acquisition of the NMR spectrum of a selected ROI. From the ${ }^{1} \mathrm{H}-\mathrm{NMR}$ and NIR spectra, it was clarified that the lower layer was composed of water, oil, and the hydrophilic organic solvent. According to the manufacturer's information, a considerable mass of glycerin is incorporated into the moisturizing cream, and this is highly soluble in water; therefore, the hydrophilic organic solvent in the lower layer was probably glycerin. There was an oil layer in the upper and middle layers, and although it was difficult to identify the oils in the upper and middle layers from the spectroscopic methods employed, valuable information was obtained from the quantitative $T_{2}$ map. The $T_{2}$ relaxation time has been shown to be sensitive to the viscosity of an oil, and a correlation between the $T_{2}$ relaxation time of crude oils and $n$-alkanes has been reported, ${ }^{19-22)}$ in that their relaxation time increased with decreasing viscosity. As shown in the histogram in Fig. $5 \mathrm{~b}$, the $T_{2}$ value of the upper layer was much greater than that of the middle layer, indicating that oils in the upper layer were more fluid than those in the middle layer. From the manufacturer's information, the moisturizing cream used contained various oil components, including liquid paraffin, squalene, ceresin, white petrolatum, beeswax, and glycerin fatty acid ester. Among these components, liquid paraffin and squalene have low melting points and are liquids at room temperature, and so these fluid oils were thought to be the main components of the upper layer.

As well as the water and oil, the distribution of active pharmaceutical ingredient (API) was assumed to be heterogeneous after phase separation. To confirm this, we measured the concentration of API in the phase-separated layers of the centrifuged samples. In this experiment, CLB ointment was employed as a model steroid ointment. The sample was prepared by mixing moisturizing cream with the CLB ointment instead of white petrolatum. In preliminary experiments, the same results as those shown in Fig. 1 were obtained. The mixed preparation separated into three distinct layers after centrifugation, and a heterogeneous distribution of water and oil was also observed (see Supplementary Material, Fig. S1).

As anticipated, a heterogeneous CLB distribution was observed in the phase-separated sample. The concentration of CLB in the lower layer was more than double those in the upper and middle layers. The CLB ointment did not completely dissolve in the ointment base, and a considerable amount of CLB existed in a crystalline form in the ointment. ${ }^{9)}$ The moisturizing cream was supposed to have a high capacity to dissolve CLB because it contained some components that are miscible with CLB, including oils, surfactants, and organic solvents. Taken together, when the CLB ointment is mixed with the moisturizing cream, the CLB crystals in the ointment probably dissolve into the components of the moisturizing cream.

The different state of CLB in the mixed preparation compared with the original ointment may be accompanied by a different pharmacological effect of CLB, because the state of CLB in the preparation is thought to significantly influence the penetration rate of CLB through the skin. The equivalent CLB concentrations in the upper and middle layers were acceptable, because each oil has hydrocarbon groups by its nature, and thus the same fundamental chemical structure probably results in the similar solubility of CLB.

In practice, a combination of moisturizing cream and CLB ointment is usually prescribed to patients as a mixed preparation. Regarding the physical stability of these mixed preparations, although there is still much room for further investiga- 
tion, the incompatibly of the components of these preparations is valuable information for their clinical use.

\section{Conclusion}

This study confirmed that the physical stability of mixed preparations is substantially decreased by mixing moisturizing cream with an ointment base. The MRI technique contributed substantially to our gaining comprehensive knowledge about the compatibility of moisturizing creams and ointment bases. It is also worth noting that using MRS and CSS images is an attractive MR technique for the component analysis of pharmaceuticals. We believe that our findings offer profound insights into the clinical use of externally applied pharmaceuticals.

Acknowledgments This study was supported by a Grantin-Aid for Scientific Research from the Japan Society for the Promotion of Science (\#25460045), the research foundation for pharmaceutical sciences, and MEXT-Supported Program for the Strategic Research Foundation at Private Universities (S1411019). The authors thank PerkinElmer Japan Co., Ltd. for their technical support.

Conflict of Interest The authors declare no conflict of interest.

Supplementary Materials The online version of this article contains supplementary materials.

\section{References}

1) Aulton M. E., Taylor K. M. G., “Aulton's Pharmaceutics: The Design and Manufacture of Medicines," Elsevier, Edinburgh, U.K., 2013.

2) McClements D. J., Crit. Rev. Food Sci. Nutr., 47, 611-649 (2007).

3) Hessien M., Singh N., Kim C., Prouzet E., Langmuir, 27, 22992307 (2011).
4) Shukla A., Janich M., Jahn K., Krause A., Kiselev M. A., Neubert R. H. H., Pharm. Res., 19, 881-886 (2002).

5) Shukla A., Krause A., Neubert R. H. H., J. Pharm. Pharmacol., 55, 741-748 (2003).

6) Song M. G., Jho S. H., Kim J. Y., Kim J. D., J. Colloid Interface Sci., 230, 213-215 (2000).

7) Onuki Y., Horita A., Kuribayashi H., Okuno Y., Obata Y., Takayama K., Drug Dev. Ind. Pharm., 40, 937-943 (2014).

8) Nishikawa M., Onuki Y., Okuno Y., Takayama K., Chem. Pharm. Bull., 59, 332-337 (2011).

9) Yamamoto Y., Fukami T., Koide T., Onuki Y., Suzuki T., Metori K., Katori N., Hiyama Y., Tomono K., Int. J. Pharm., 463, 62-67 (2014).

10) Haase A., Frahm J., Hanicke W., Matthaei D., Phys. Med. Biol., 30, 341-344 (1985).

11) Hashemi R. H., Bradley W. G. Jr., Lisanti C. J., "MRI: The Basics," Lippincott Williams \& Wilkins, Philadelphia, 2010.

12) Tan B. L., Reddy N., Sarafis V., Beattie G. A. C., Spooner-Hart R., HortScience, 40, 720-725 (2005).

13) Peng X. G., Ju S., Fang F., Wang Y., Fang K., Cui X., Liu G., Li P., Mao H., Teng G. J., Am. J. Physiol. Endocrinol. Metab., 304, E160E167 (2013)

14) Peng X. G., Bai Y. Y., Fang F., Wang X. Y., Mao H., Teng G. J., Ju S., Radiology, 269, 748-757 (2013).

15) Gersbach P. V., Reddy N., Ann. Bot., 90, 253-257 (2002).

16) Halloin J. M., Cooper T. G., Potchen E. J., Thompson T. E., J. Am. Oil Chem. Soc., 70, 1259-1262 (1993).

17) Rumpel H., Pope J. M., Magn. Reson. Imaging, 10, 187-194 (1992).

18) Tse T. Y., Spanswick R. M., Jelinski L. W., Protoplasma, 194, 54-62 (1996).

19) Hirasaki G. J., Lo S. W., Zhang Y., Magn. Reson. Imaging, 21, 269-277 (2003).

20) Morriss C. E., Freedman R., Straley C., Johnston M., Vinegar H. J., Tutunjian P. N., Log Anal., 38, 44-59 (1997).

21) Straley C., Rossini D., Vinegar H., Tutunjian P., Morriss C., Log Anal., 38, 84-93 (1997).

22) LaTorraca G. A., Dunn K. J., Webber P. R., Carlson R. M., Magn. Reson. Imaging, 16, 659-662 (1998). 